\section{PTU-086 VIRTUAL BIOLOGICS CLINIC FOR IBD PATIENTS- IMPROVING PATIENT AND SERVICE EXPERIENCE}

Emily Creed*, Katie Clark. St Helens and Knowlsey Nhs Trust, Prescot, UK

\subsection{6/gutjnl-2019-BSGAbstracts.445}

Introduction Increasing demand on outpatient services, increased use of high cost drugs and the desire to involve patients more in their own care led to the introduction of a virtual multidisciplinary clinic for patients with inflammatory bowel disease (IBD) at a large district general hospital. The key drivers were to standardise and enhance pre-biologic screening and monitoring, to collect accurate, real-time data to enhance national audit, to realise service and cost efficiencies yet still provide a service that remains acceptable to our population.

Methods We sent questionnaire to our patients, gauging current opinion on the service and their thoughts on a virtual clinic (would it meet needs, would they accept this type of review) and achieved a $45 \%$ response rate. We also performed a pilot clinic of 20 randomly selected patients with IBD on biologic drugs and collected data related to screening, and whether a virtual clinic would work within the broader goal of a treat-to-target strategy for these patients. From the positive response, the clinic was established comprising a consultant Gastroenterologist, IBD nurse and pharmacist.

Results The clinic has been established for almost 12 months. In that time:

1. We have established a robust, easy to maintain database that directly links to the data required for the IBD Registry. The database can also be utilised by our research team to identify appropriate patients for clinical trials.

- 50 patients have had their biologic treatment stopped due to biochemical remission with only 3 out of those requiring further treatment.

- 34 patents have had their medication switched in a timely manner due to early identification of non or lack of response (mean time 35 days)

- - Cost savings have returned to the CCG

- Patients report they are 'highly satisfied' with the service as they know their treatment is under regular review without the need for additional hospital visits.

Conclusions The virtual biologics clinic provides a robust, standardised review for all IBD patients on high cost drugs without impacting on already overstretched outpatient clinic capacity. In addition to significant cost savings to the CCG, improved data collection and participation in national audit; the service is acceptable to the patients and is now an integral part of the IBD service.

\section{PTU-087 SPECIALIST MDT CLINIC MANAGEMENT IMPROVES ACCEPTANCE RATES AND POST-TRANSPLANT RELAPSE IN PATIENTS WITH ARLD}

${ }^{1}$ Michael Ding* ${ }^{1}$ Charlotte Parker, ${ }^{1}$ Jennifer Towey, ${ }^{1}$ Matthew Harborne, ${ }^{1}$ Chiemelie Ngonadi, 'Malik Magrabi, 'Laurence Hopkins, ${ }^{2}$ Anya Mohideen, ${ }^{2}$ Abbie Harrison, ${ }^{2}$ Jon Catling, ${ }^{1,3}$ Neil Rajoriya, ${ }^{1,3}$ Andrew Holt. ${ }^{1}$ The Liver Unit, Queen Elizabeth Hospital, UK; ${ }^{2}$ The School of Psychology, University of Birmingham, UKi ${ }^{3}$ Both authors contributed equally

10.1136/gutjil-2019-BSGAbstracts.446
Introduction Alcohol-related Liver Disease (ArLD) is the most common indication for liver transplantation (LT) in the UK $(30 \%)$ but many patients are deemed physically or psychologically unsuitable at the time of LT assessment and post-LT alcohol relapse rates remain high (1-2\% Kawaguchi et al. 2014). A specialist high intensity ArLD clinic was established to optimise patient's pre-LT assessment from a medical, addiction, psychological and nutritional perspective and evaluate high risk patients assessed for LT within a specialist MDT clinic environment. The clinic comprises of 2 Transplant Hepatologists, addiction psychiatry specialists, liver dietician \& researchers.

The aim was to review the impact of the high intensity clinic programme in patients transplanted for complications of ArLD cirrhosis.

Methods Data was reviewed retrospectively from all patients attending clinic from inception, 25/5/2016- 31/9/2018. Electronic notes and alcohol addictions/transplant databases were interrogated. Patients were contacted anonymously (independent from health practitioners) by Psychology researchers for follow-up psychological data.

Results 261 patients were seen, 247 (seen pre-LT) were included for analysis (173M: 74F), mean age 54y (SD $\pm 19.65)$, UKELD=53 (SD \pm 5.63$) .124$ (50\%) were presented in the transplant listing meeting, 71 (57.25\%) accepted. 46 underwent LT at time of analysis, median waiting list time $=39 \mathrm{~d}$ [range 3-373]. 11/247 attendees (4\%) had documented relapse within the programme pre-LT.

$54 / 247(21.9 \%)$ were referred to the high intensity clinic after being turned down by the transplant listing meeting, considered physically or psychologically unsuitable. Of these, $59.3 \%$ were re-presented after further intervention and subsequently listed. Of all attendees, 54 deaths (21.9\%) were recorded on follow-up ( 2 on the LT waiting list \& 4 post LT).

Follow-up questionnaire completion rate was 78\% (32/41) demonstrating high levels of patient engagement. Patient satisfaction with the clinic was high. Median follow-up post-LT was 13 mo [range -8.4]). Post-transplant relapse in this high risk group managed in the high intensity clinic was $0 / 46$ $(0 \%)$-vs $6 / 49(12.2 \%, \mathrm{p}<0.05)$ in a historical post-LT ArLD group (201-9) not seen in the high intensity clinic $(n=49$ contacted from 68 transplanted, 72\%).

Conclusion The High Intensity MDT clinic is an effective clinical environment for treating high risk patients and managing their physical and psychological risk factors. Specialist addiction-focussed MDT management enables patients who were initially deemed unsuitable for transplantation to be listed for surgery and is shown to significantly reduce short term relapse in this cohort of patients.

\section{PTU-088 DAY CASE ELECTIVE PARACENTESIS}

Hannah Dix*, Miss Heather Cracknell, Elaine Henry, Morag Barron. NHS Tayside, Perthshire, UK

\subsection{6/gutjnl-2019-BSGAbstracts.447}

Introduction Increasing prevalence of chronic liver disease has caused demand for elective paracentesis in refractory ascites to exceed capacity in NHS Tayside, with patients diverting to acute care areas. Assessing the current elective care pathway 